\title{
THE GAP OF THE GRAPH OF A LINEAR TRANSFORMATION
}

\section{JAVAD FAGHIH-HABIBI AND RALPH HOLLINGSWORTH}

\begin{abstract}
Assume that $A$ is a linear transformation from $C^{n}$ into $C^{m}$. The Gap of the graph of $A$ is

$$
\theta\left(A_{g}\right)=\frac{\|A\|}{\sqrt{1+\|A\|^{2}}} .
$$

Here $\|A\|$ is the operator norm of $A$.

This is an extention of the result in [2], in which the first author used another method to prove for the case of $m=n$.
\end{abstract}

\section{Introduction}

Consider $C^{k}$ with the usual scalar product and the corresponding norm. Let $S_{i}$ be the unit sphere in the subspace $M_{i} \subseteq C^{k}$ for $i=1,2$.

The gap between $M_{1}$ and $M_{2}$ is defined as

$$
\theta\left(M_{1}, M_{2}\right)=\max \left\{\sup _{x \in S_{1}} d\left(x, M_{2}\right), \sup _{x \in S_{2}} d\left(x, M_{2}\right)\right\}
$$

where $d(x, M)=\inf _{t \in M}\|x-t\|$ is the distance of $x$ from the set $M \subseteq C^{k}$.

The gap is being used to determine stable invariant subspaces of a linear transformation which is important from the point of view of numerical computations.

See Gohberg, Lancaster and Rodman [1].

Let $A: C^{n} \longrightarrow C^{m}$ be a linear transformation. We assume that $A$ is given by an $m \times n$ matrix with respect to the standard orthonormal bases. Let

$$
G_{A}=\left\{\left(\begin{array}{c}
x \\
A x
\end{array}\right): x \in C^{n}\right\} \subset C^{n} \oplus C^{m}
$$

be the graph of $A$. The norm of $A$ is

$$
\|A\|=\sup _{\|x\|=1}\|A x\| .
$$

Received December 17, 1993. 
The case $m=n$ has been dealt with in [2] with different proof.

\section{The Gap of the Graph of $A$}

The gap of the graph of $A$ is the gap between two subspaces $G_{A}$ and $G_{0}, \theta\left(G_{A}, G_{0}\right)$, which we denote by $\theta\left(A_{g}\right)$. Set

$$
f(x, y)=d\left(\left(\begin{array}{l}
x \\
0
\end{array}\right),\left(\begin{array}{c}
y \\
A y
\end{array}\right)\right)=\sqrt{\|x-y\|^{2}+\|A y\|^{2}} .
$$

Then

$$
\theta\left(A_{g}\right)=\max \left\{\sup _{\|x\|=1}\left[\inf _{y} f(x, y)\right], \sup _{\|y\|^{2}+\|A y\|^{2}=1}\left[\inf _{x} f(x, y)\right]\right\} .
$$

Note that

$$
f(x, y) \geq \sqrt{\|x\|^{2}+\|y\|^{2}+\|A y\|^{2}-2\|x\| \quad\|y\|}
$$

and the equality sign holds if $y=c x$ for some $c>0$.

Choose $y=c x$ for some $c>0$, we have (assuming $\|x\|=1$ )

$$
f(x, y)=\sqrt{\left(1+\|A x\|^{2}\right) c^{2}-2 c+1}
$$

Set $c=\frac{1}{1+\|A x\|^{2}}$, to obtain

$$
\inf _{y} f(x, y)=\frac{\|A x\|}{\sqrt{1+\|A x\|^{2}}}
$$

Therefore

$$
\sup _{\|x\|=1}\left[\inf _{y} f(x, y)\right]=\sup _{\|x\|=1} \frac{\|A x\|}{\sqrt{1+\|A x\|^{2}}} .
$$

Since $\frac{t}{\sqrt{1+t^{2}}}$ is an increasing function for $t \geq 0$, and $\|A\|=\sup _{\|x\|=1}\|A x\|$, we have

$$
\sup _{\|x\|=1}\left[\inf _{y} f(x, y)\right]=\frac{\|A\|}{\sqrt{1+\|A\|^{2}}}
$$

Now consider $y \in C^{n}$ with $\|y\|^{2}+\|A y\|^{2}=1$. We see that

$$
f(x, y) \geq \sqrt{\|x\|^{2}-2\|x\|\|y\|+1} .
$$

Set $x=c y$ for some $c>0$ to get

$$
f(x, y)=\sqrt{\|y\|^{2} c^{2}-2 c\|y\|^{2}+1} .
$$


Choosing $c=1$, we have

$$
\inf _{x} f(x, y)=\sqrt{1-\|y\|^{2}}=\|A y\|
$$

Setting $y=k z$, with $\|z\|=1$, we have $\sup \left\{\|A y\|:\|y\|^{2}+\|A y\|^{2}=1\right\}=\sup \{|k|$ $\left.\|A z\|:|k|^{2}\left(1+\|A z\|^{2}\right)=1\right\}=\sup _{\|z\|=1} \frac{\|A z\|}{\sqrt{1+\|A z\|^{2}}}$.

Sicne $\sup _{\|z\|=1}\|A z\|=\|A\|$, and $\frac{t}{\sqrt{1+t^{2}}}$ is an increasing continuous function of $t \geq 0$, we have

$$
\begin{aligned}
& \sup \frac{\|A z\|}{\sqrt{1+\|A z\|^{2}}}=\frac{\|A\|}{\sqrt{1+\|A\|^{2}}} \text {, and } \\
& \sup _{\|y\|^{2}+\|A y\|^{2}=1}\left[\inf _{x} f(x, y)\right]=\frac{\|A\|}{\sqrt{1+\|A\|^{2}}} .
\end{aligned}
$$

Therefore

$$
\theta\left(A_{g}\right)=\frac{\|A\|}{\sqrt{1+\|A\|^{2}}}
$$

\section{References}

[1] I. Gohberg, P. Lancaster and L. Rodman., Invariant Subspaces of Matrices with Applications, John Wiley and Sons, New York, 1986.

[2] J. F. Habibi, "The gap of the graph of a matrix," Linear Algebra Appl., 186 (1993), 55-57.

Department of Mathematics and Computer Science, Muskingum College, New Concord, Ohio 43762, U.S.A. 